\title{
Teratogenic effects of aqueous extract of Ficus glomerata leaf during embryonic development in zebrafish (Danio rerio)
}

\author{
Abusufyan Shaikh ${ }^{1 *}$, Kalidas Kohale², Mohammed Ibrahim³, Mohib Khan ${ }^{4}$ \\ ${ }^{1}$ School of Pharmacy, Anjuman-I-Islam's Kalsekar Technical Campus, New Panvel, Maharashtra, affiliated to Mumbai University, Mumbai, India. \\ ${ }^{2}$ Department of Biological Sciences, Tata Institute of Fundamental Research, Mumbai, India. \\ ${ }^{3}$ PNR College of Pharmacy, Hyderabad, India. \\ ${ }^{4}$ Oriental College of Pharmacy, Mumbai, India.
}

\begin{tabular}{l}
\hline ARTICLE INFO \\
\hline Received on: $25 / 01 / 2019$ \\
Accepted on: $11 / 03 / 2019$ \\
Available online: $08 / 05 / 2019$ \\
\\
\hline Key words: \\
Ficus glomerata, toxicity test, \\
teratogenesis, embryogenesis.
\end{tabular}

\section{INTRODUCTION}

Plants have been a source of medicine over centuries and been part of the traditional healing practices. Ficus glomerata (FG) leaf has been traditionally used for the treatment of bronchitis, bowel syndrome, wound healing, and piles (Joseph and Raj, 2010). The leaves of FG contained active constituents such as kampferol, rutin, arabinose, bergapten, psoralenes, ficusin, and coumarin (Baruah and Gohain, 1992; Deraniyagala et al., 1998). Scientific study on aqueous extract of FG leaf (AEFG) revealed its antioxidant (Abusufyan et al., 2018; Eshwarappa et al., 2015), anthelmintic (Divakar et al., 2017), antidiabetic (Divyash et al., 2017a), and antiulcer activity (Divyash et al., 2017b). However, its teratogenic effect should be investigated to obtain safety

${ }^{*}$ Corresponding Author

Abusufyan Shaikh, School of Pharmacy, Anjuman-I-Islam's Kalsekar Technical Campus, New Panvel, Mumbai University, Mumbai, India. Email: abusufyan.shaikh@aiktc.ac.in characteristics of extract prior to its development as a potential pharmacological agent. Despite possessing several therapeutic benefits by the medicinal plants, some of their phytoconstituents have been known to cause potent mutagenicity, carcinogenicity, and teratogenicity (Akintonwa et al., 2009). Teratogens are any substances responsible for the formation of anatomical abnormalities or defects in the developing embryos.

Developing embryos of zebrafish is transparent which permits imaging of the internal organs (Rajaretinam et al., 2015). In addition, this model system has attracted the attention of the researchers for the study of the teratogenic activity of the drugs due to external fertilization, development, and rapid organogenesis of embryonic zebrafish. The present study was undertaken to investigate $\mathrm{LC}_{50}$ value and effects of $\mathrm{AEFG}$ on embryonic development using zebrafish embryo as a model system. 


\section{MATERIALS AND METHODS}

\section{Preparation of Ficus glomerata leaf extract}

The leaves of FG were collected from Sindhudurg district of Maharashtra, India and identified with authentication no. 15-023 by botanist Dr. A. S. Upadhye of Agharkar Research Institute, Pune, India. The powdered leaf material was extracted by hot water extraction and concentrated by using rotary evaporator.

\section{Preliminary phytochemical screening}

The AEFG extract of FG was characterized by its preliminary qualitative phytochemical screening for alkaloids, amino acids, flavonoids, glycosides, phenolics, tannins, triterpenoids, and steroids by using the standard methods (Khandelwal, 2013).

\section{Zebrafish maintenance and embryos collection}

Adult zebrafish were collected from local pet shop located in Mumbai, India. These fish showed characteristics as described by Wixon (2000). All fish were maintained, breed and eggs were collected according to standard procedure (Dulay et al., 2012). The research protocol was approved by the Institutional Animal Ethics Committee with reference No. AIKTC/SoP/ IAEC/2017/01.

\section{Zebrafish embryo toxicity test}

Zebrafish embryo toxicity test of AEFG was studied by using zebrafish embryos with test time of 96 hours in accordance with Organization for Economic Co-operation and Development (OECD) TG No. 236, 2013. The procedures were adopted with slight modification from several standard methods studied earlier (Indra et al., 2018; Ingrid et al., 2019; OECD, 2013; Thangavel etal., 2012). After initial range-finding experiments, five concentrations of AEFG 125, 250, 500, 1,000, and 2,000 ppm were selected as the final exposure concentrations. Three replicate treatment groups $(3 \times 30$ embryos $)$ were assayed in 96 well microplates incubated at $27.0^{\circ} \mathrm{C} \pm 1.0^{\circ} \mathrm{C}$. Evaluation was conducted by using parameters of lethality, i.e., coagulation, non-detachment of the tail, somite disruption, and lack of heart-beat. $\mathrm{LC}_{50}$ value was calculated using probit analysis in MS-Excel.

\section{Hatchability}

A zebrafish embryo is considered as hatched when the entire body of larvae from tail to head is out of the chorion. Percent hatchability after 72 hours post-treatment exposure (hpte) was calculated using the formula:

$$
\% \text { Hatchability }=\frac{\text { No. of hatched embryos }}{\text { Initial no. of embryos }} * 100
$$

\section{Heartbeat rates and total body length of zebrafish}

Numbers of heartbeat per minute of anesthetized zebrafish embryos after 72 hpte were recorded under Labomed digital microscope. For determining the total body length, images of anesthetized zebrafish larvae after 96 hpte were taken and measured by using image $\mathrm{J}$ software.

\section{Study of developmental abnormalities in zebrafish embryos}

Developmental morphological abnormalities were studied by using parameters such as delayed growth, limited movement, abnormal head-trunk angle, scoliosis/flexure, and yolk sac edema (Nagel, 2002). These effects were examined and images were taken after 24, 48, and 72 hpte by using a Labomed digital microscope.

\section{Statistical analysis}

Data were analyzed by one-way analysis of variance, followed by post-hoc test, paired sample $t$-test, and descriptive statistic by using JASP 0.9.1.0 software.

\section{RESULTS AND DISCUSSION}

Preliminary qualitative phytochemical screening was performed to investigate the nature of chemical constituents present in AEFG. The results of preliminary phytochemical screening are listed in Table 1. Alkaloids, flavonoids, phenolics, and saponins were identified in AEFG.

The laboratory zebrafish is an established animal model for in vivo drug discovery and toxicology studies (Sipes et al., 2011; Strahle and Grabher, 2010; Zon and Peterson, 2005). Fish embryo toxicity test of AEFG was studied on zebrafish embryos exposed to different concentrations of test solution for 96 hours. This test was done to determine the $\mathrm{LC}_{50}$ value of AEFG. $\mathrm{LC}_{50}$ value is the concentration of extract which causes death in $50 \%$ of test animals. The zebrafish embryo was considered as dead if it showed any one parameter of lethality, i.e., coagulation, non-detachment of the tail, somite disruption, and lack of heartbeat. No mortality was observed in embryos exposed to $0-100 \mathrm{ppm}$ concentrations of extract. The data of percentage mortality after 96 hpte with selected concentration of test solution were analyzed by probit analysis with $95 \%$ confidence interval and $5 \%$ alpha. The $\mathrm{LC}_{50}$ value of AEFG was found to be 239.88 ppm (Fig. 1). Higher $\mathrm{LC}_{50}$ values indicate less toxicity of test drug as greater concentrations are required to produce 50\% mortality in organisms (Basha and Rani, 2003). As per OECD guidelines, toxicity of pollutants against zebrafish is categorized as harmful $\left(10 \mathrm{mg} / 1<\mathrm{LC}_{50}<100 \mathrm{mg} / \mathrm{l}\right)$, toxic $(1 \mathrm{mg} / \mathrm{l}$ $\left.<\mathrm{LC}_{50}<10 \mathrm{mg} / \mathrm{l}\right)$, and highly toxic $\left(\mathrm{LC}_{50}<1 \mathrm{mg} / \mathrm{l}\right)$. Based on this, AEFG extract was found to be relatively safe. Previous study also suggested that AEFG extract was found to be safe in acute oral toxicity test with a single oral dose of $2,000 \mathrm{mg} / \mathrm{kg}$ in Wistar rats (Divyash et al., 2017b).

Table 1. Phytochemical screening of AEFG.

\begin{tabular}{lc}
\hline Chemical constituents & AEFG \\
\hline Alkaloids & + \\
Amino acids & - \\
Flavonoids & + \\
Glycosides & - \\
Phenolics & + \\
Saponins & + \\
Tannins & - \\
Triterpenoids & - \\
Steroids & - \\
\hline$+=$ positive, $-=$ negative. &
\end{tabular}




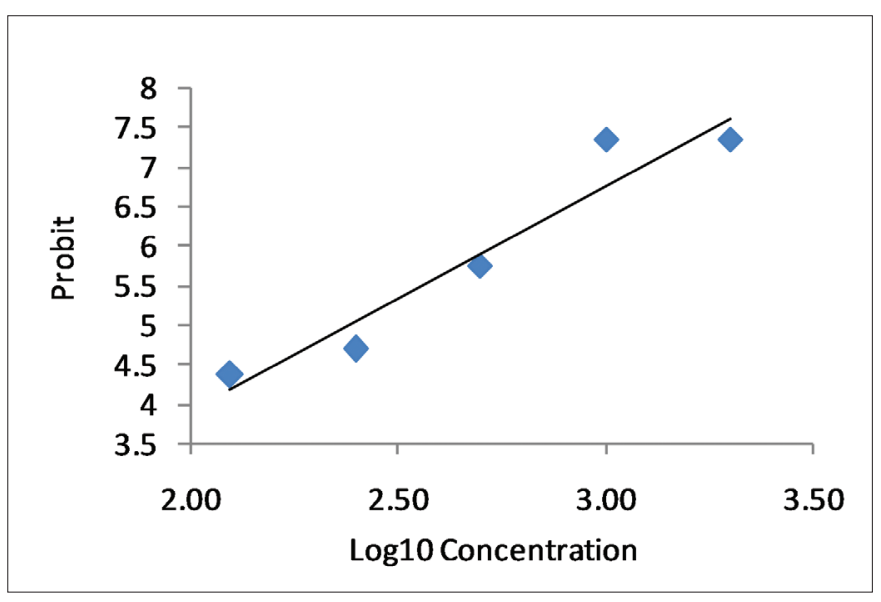

Figure 1. The linear regression curve of $\log 10$ Concentration versus probit of AEFG on zebrafish embryos. $y=2.850 * X-1.803 . R^{2}=0.92592$.

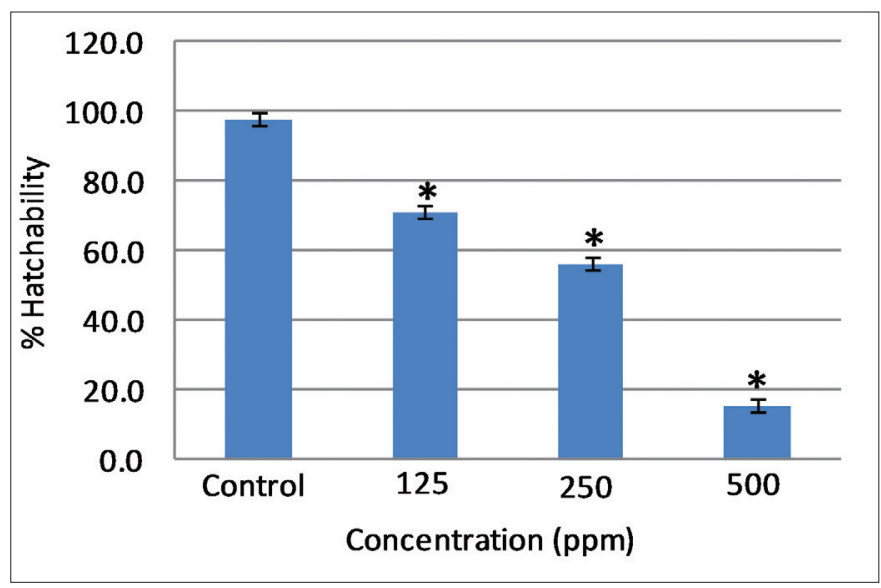

Figure 2. Effect of different concentrations of AEFG on \% hatchability in zebrafish embryo. Values are expressed as mean $\pm \mathrm{SD}\left(n=30\right.$ embryos); ${ }^{*} p<$ 0.05 when compared to corresponding control.

Hatching was completed after 72 hours post fertilization in control embryos. However, a significant decrease $(p<0.05)$ in the hatching rate was observed at all selected concentrations of AEFG as compared to control (Fig. 2). Low hatchability and high mortality rate at a high concentration of AEFG could be attributed to the delayed embryogenesis of zebrafish embryos and can, therefore, be one of the important aspects of its sublethal properties. It has been reported that the slimy coating around embryos due to the high concentration of extract might contribute to the death and decrease in overall hatchability (Asharani et al., 2008). Similar slimy coating was seen around embryos with a high concentration of AEFG.

Heartbeat is an important sublethal endpoint which is routinely measured in zebrafish embryos as an index of toxicity. Control embryos show normal heartbeat after 96 hours post fertilization. Significant dose-dependent decrease $(p<0.001)$ in the heart rate was observed at selected concentrations of AEFG as compared to control (Fig. 3). Impaired cardiac functioning in the underdeveloped heart may induce an abnormal heartbeat and failure of blood circulation with subsequent retardation of body

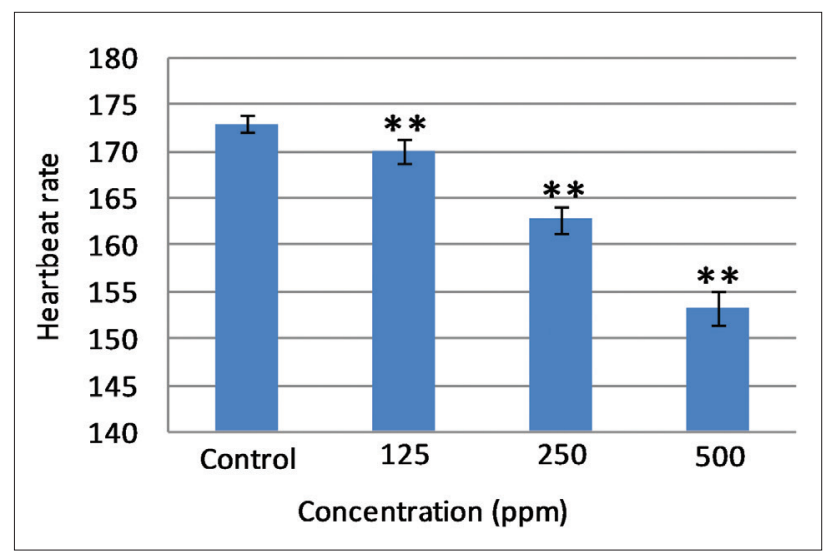

Figure 3. Effect of different concentrations of AEFG on heartbeat rate in zebrafish embryo. Values are expressed as mean $\pm \operatorname{SD}(n=10$ embryos); ${ }^{* *} p<0.001$ when compared to corresponding control.

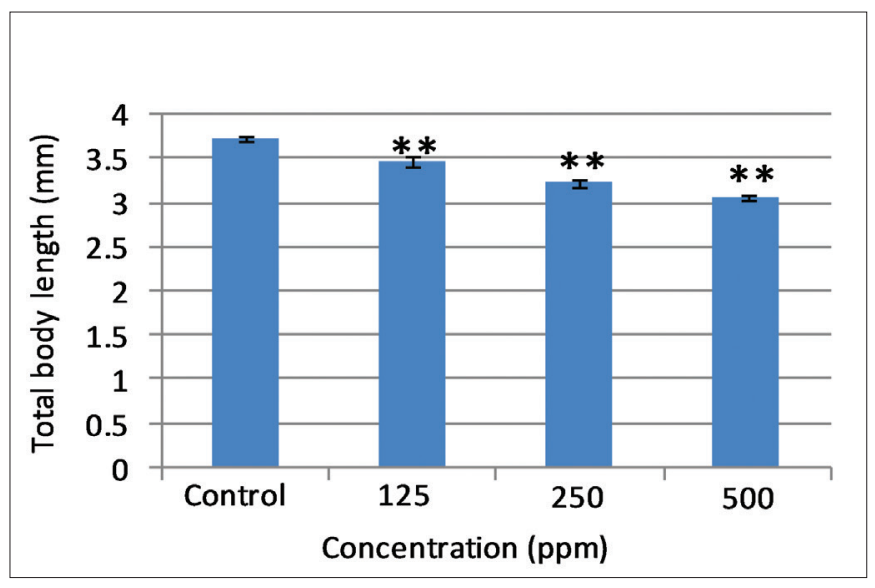

Figure 4. Effect of different concentrations of AEFG on total body length $(\mathrm{mm})$ in zebrafish embryo. Values are expressed as mean $\pm \mathrm{SD}(n=10$ embryos); ** $p<0.001$ when compared to corresponding control.

growth caused by deficiency of nutrients (Majewski et al., 2017). Control larvae showed total mean body length of $3.72 \pm 0.035$ $\mathrm{mm}$ which is in accordance with the length of normal larvae mentioned in zebrafish developmental staging series (ZFIN, 2018). A significant decrease $(p<0.001)$ in total body length of zebrafish larvae was observed in selected concentrations of AEFG as compared to control (Fig. 4). Concentration-dependent decrease in total body length in AEFG treated zebrafish larvae might be due to the failure of blood circulation and nutritional deficiency associated with a reduction of heartbeat rate. Therefore, it should be noted that the developing zebrafish heart might be a potential target of the high concentration of AEFG toxicity.

Developmental effects of the different concentrations of the AEFG were studied after 24, 48, and 72 hpte and presented in Tables 2 and 3. Active embryos with spontaneous movement and completely detached tail were observed at 0 and $125 \mathrm{ppm}$ concentrations of AEFG. However, high concentration (250, $500,1,000$, and 2,000 ppm) of AEFG extract showed delayed growth, limited movement, and slightly detached tail at 24 and 48 hpte (Table 3, A1 to B6). Yolk sac edema, scoliosis/flexure, 
Table 2. Teratogenic effects of AEFG in zebrafish embryos.

\begin{tabular}{|c|c|c|c|c|c|c|}
\hline $\begin{array}{c}\text { Concentration } \\
(\mathrm{ppm})\end{array}$ & Delayed growth & $\begin{array}{c}\text { Limited } \\
\text { movement }\end{array}$ & $\begin{array}{c}\text { Slightly } \\
\text { detached tail }\end{array}$ & $\begin{array}{c}\text { Abnormal } \\
\text { head-trunk angle }\end{array}$ & $\begin{array}{l}\text { Scoliosis/ } \\
\text { Flexure }\end{array}$ & Yolk sacedema \\
\hline 125 & - & - & - & - & - & - \\
\hline 250 & + & + & + & - & - & + \\
\hline 500 & + & + & + & + & + & + \\
\hline 1,000 & + & + & + & NA & NA & NA \\
\hline 2,000 & + & + & + & NA & NA & NA \\
\hline
\end{tabular}

Table 3. Representative images of teratogenic effects of AEFG in zebrafish embryos.

\begin{tabular}{|c|c|c|c|}
\hline Concentrations ppm & 24 hpte & 48 hpte & 72 hpte \\
\hline 0 & & & \\
\hline 125 & & & \\
\hline 250 & & & \\
\hline 500 & & & \\
\hline 1,000 & & & \\
\hline 2,000 & & & \\
\hline
\end{tabular}

(A3 to C6): Indicate delayed embryogenesis; (C3, C4): White arrow showing yolk sac edema at 250, $500 \mathrm{ppm}$ after $72 \mathrm{hpte}$ of AEFG; (C4): Red arrow showing scoliosis/flexure at $500 \mathrm{ppm}$ after $72 \mathrm{hpte}$ of AEFG; (C4): Black arrow showing abnormal head-trunk angle at 500 ppm after 72 hpte of AEFG; (C5,

C6): Blue arrow showing coagulation of zebrafish embryos at 1,000, 2,000 ppm after 72 hpte of AEFG.

and abnormal head-trunk angle were the three most commonly occurring morphological changes observed at 250 and $500 \mathrm{ppm}$ concentration after 72 hpte of AEFG (Table 3, C3 and C4).

This study is the first investigation of AEFG extract on developmental toxicity in zebrafish embryos. Previous studies suggested that chemicals and drugs have similar toxicological effect in developing embryos (Driever et al., 1996; Lam et al., 2005). Preliminary qualitative phytochemical investigation showed the presence of alkaloids, flavonoids, phenolics, and saponins which have been reported for their cytotoxic actions (Romagosa et al., 2016). This strong cytotoxic activity can be significantly considered for the future evaluation of its anticancer, antitumor, and apoptotic properties.

\section{CONCLUSION}

Based on aquatic toxicity classification of OECD, AEFG was classified as safe. However, high concentration of AEFG showed developmental toxicity in zebrafish embryos 
such as low percentage hatchability, decreased heartbeat rate, decreased total body length, delayed growth, slightly detached tail, abnormal head-trunk angle, scoliosis/flexure, yolk sac edema, and coagulation of embryos. The results showed a significant concentration-response relationship between toxicity endpoints and AEFG concentration. However, the mechanism of action of AEFG constituents and investigation of individual compound responsible for the described malformations are needed to be studied.

\section{ACKNOWLEDGMENTS}

The author wishes to thank Dr. Abdul Razak Honnutagi, Director, AI-Kalsekar Technical Campus and Dr. Shariq Syed, Dean, School of Pharmacy, AI-Kalsekar Technical Campus for providing facilities to carry out this work.

\section{FINANCIAL SUPPORT AND SPONSORSHIP}

None.

\section{CONFLICT OF INTERESTS}

The authors declare that they have no conflicts of interest.

\section{REFERENCE}

Abusufyan S, Ibrahim M, Mohib K. Comparative in vitro antidiabetic and antioxidant activity of various extracts of Ficus species. Pharmacog J, 2018; 10(2):349-54.

Akintonwa A, Awodele O, Afolayan G, Coker HA. Mutagenic screening of some commonly used medicinal plants in Nigeria. J Ethnopharmacol, 2009; 125:461-70.

Asharani PV, Lian Wu Y, Gong Z, Valiyaveettil S. Toxicity of silver nanoparticles in zebrafish models. Nanotechnology, 2008; 19(25):255102.

Available via https://zfin.org/zf_info/zfbook/stages/index.html (Accessed 25 December 2018).

Baruah KK, Gohain AK. Chemical composition and nutritive value of Dimaru (Ficus glomerata Roxb.) leaves. Indian J Anim Nutr, 1992; 9:107-8.

Basha PS, Rani AU. Cadmium-induced antioxidant defense mechanism in fresh water teleost Oreochromis mossambicus (tilapia). Ecotoxicol Environ Saf, 2003; 56(2):218-21.

Romagosa CMR, David ES, Dulay RMR. Embryo-toxic and teratogenic effects of Tinospora cordifolia leaves and bark extracts in zebrafish (Danio rerio) embryos. Asian J Plant Sci Res, 2016; 6(2):37-41.

Deraniyagala SA, Wijesundera RLC, Weerasena OVDSJ. Antifungal activity of Ficus racemosa leaf extract and isolation of the active compound. J Nat Sci Council Sri Lanka, 1998; 26:19-26.

Divakar K, Srinivasa U, Prabhavathi J, Madhuri AN, Bhargavi Y, Anjali YV, Swamy GM. Anthelmintic activity of leaves of Ficus racemosa. Int J Pharm Sci Res, 2017; 5(2):12-3.

Divyash S, Amresh G, Pushpendra KS, Shobha S, Ravindra PS. Phytochemical and in vivo pharmacological evaluation of various extracts of Ficus racemosa L. Int J Green Pharm, 2017a; 11(4):268-75.

Divyash S, Shobha S, Amresh G, Ravindra PS. Anti-ulcer activity of leaves and bark of Ficus racemosa linn in ethanol \& aspirin induced ulcer in rats. Int J Pharm Bio Arch, 2017b; 8(4):29-33.

Driever W, Solnica-Krezel L, Schier AF, Neuhauss SC, Malicki J, Stemple DL, Stainier DY, Zwartkruis F, Abdelilah S, Rangini Z, Belak J. A genetic screen for mutations affecting embryogenesis in zebrafish. Development, 1996; 123:37-46.
Dulay RM, Kalaw SP, Reyes RG, Alfonso N, Eguchi F. Teratogenic and toxic effects of Lingzhi or Reishi medicinal mushroom, Ganoderma lucidum (W.Curt.:Fr.) P. Karst. (Higher Basidiomycetes), on zebrafish embryo as model. Int J Med Mushrooms, 2012; 14(5):507-12.

Eshwarappa RS, Iyer S, Subaramaihha SR, Richard SA, Dhananjaya BL. Antioxidant activities of Ficus glomerata (moraceae) leaf gall extracts. Pharmacogn Res, 2015; 7(1):114-20.

Indra W, Kurnia P, Rika H, Sophi D. Ethanolic extract of pomegranate (Punica granatum L) peel: acute toxicity tests on zebrafish (Danio rerio) embryos and its toxicity prediction by in silico. J Appl Pharm Sci, 2018; 8(06):082-6.

Ingrid WTS, An RVR, Wim DC, Hilda W. Development of a screening assay to identify teratogenic and embryotoxic chemicals using the zebrafish embryo. Reprod Toxicol, 2009; 28:308-20.

Joseph B, Raj SJ. Phytopharmacological and phytochemical properties of three ficus species-an overview. Int J Pharma Bio Sci, 2010; $1: 246-53$.

Khandelwal KR. Preliminary phytochemical screening. In Vrunda KS (ed.). Practical pharmacognosy techniques and experiments. 22nd edition, Nirali Prakashan, India, pp 25.1-25.6, 2013.

Lam CS, Korzh V, Strahle U. Zebrafish embryos are susceptible to the dopaminergic neurotoxin, MPTP. Eur J Neurosci, 2005; 21:1758-62.

Majewski M, Kasica N, Jakubowski P, Podlasz P. Influence of fresh garlic (Allium sativum L.) juice on zebrafish (Danio rerio) embryos: developmental effects. J Elem, 2017; 22(2):475-86.

Nagel R. DarT: The embryo test with the zebrafish Danio rerio - a general model in ecotoxicology and toxicology. Altex, 2002; 19(1):38-48.

Organization for Economic Co-operation and Developmen (OECD). Guidelines for Testing of Chemical-Fish Embryo Acute Toxicity (FET) Test, OECD Publishing, Paris, 2013.

Rajaretinam RK, Appadurai MI, Samuel GPV. Chemical genetic effects of Sargassum wightii during embryonic development in zebrafish. Ind J Pharmacol, 2015; 47(2):195-8.

Sipes NS, Padilla S, Knudsen TB. Zebrafish: as an integrative model for twenty first century toxicity testing. Birth Defects Res C, 2011; 93(3):256-67.

Strahle U, Grabher C. The zebrafish embryo as a model for assessing off target drug effects. Dis Model Mech, 2010; 3:689-92.

Thangavel P, Moorthy G, Lonchin S. Developmental toxicity evaluation of ethanolic extract of Annona squamosa in zebrafish (Danio rerio) embryo. J Pharm Res, 2012; 5(1):277-9.

Wixon J. Danio rerio: the zebrafish. Yeast, 2000; 17:225-31.

Zon LI, Peterson RT. In vivo drug discovery in the zebrafish. Nat Rev Drug Discov, 2005; 4(1):35-44.

How to cite this article:

Shaikh A, Kohale K, Ibrahim M, Khan M. Teratogenic effects of aqueous extract of Ficus glomerata leaf during embryonic development in zebrafish (Danio rerio). J Appl Pharm Sci, 2019; 9(05):107-111. 